\title{
Is the word 'biomarker' being properly used by proteomics research in neuroscience?
}

\author{
Daniel Martins-de-Souza
}

Received: 11 January 2010/Accepted: 29 January 2010/Published online: 14 February 2010

(C) The Author(s) 2010. This article is published with open access at Springerlink.com

Lately, when researchers see an article with the word "biomarker", they feel, at least, suspicious. Sometimes, even scared and such feeling of distrust occurs mostly because the majority of the published articles in the field of global proteome analysis are revealing, for instance, differentially expressed proteins in pathologic conditions and claiming that biomarkers are being discovered. In addition to transform a differentially expressed protein in a biomarker, a long road has to be travelled.

Biomarker is defined by the United States Food and Drug Administration (FDA) as "a characteristic that is objectively measured and evaluated as an indicator of normal biologic or pathogenic processes or pharmacological responses to a therapeutic intervention" [1]. Such definition is, indeed, quite specific and very different from a differentially expressed protein discovered as potentially related to some disease in a small set of sample. Therefore, researchers are totally correct regarding their suspicions.

Searching at PubMed database (31 Dec 2009), the word "biomarker" returns 477,273 different articles that have exponentially increased in the recent years (Fig. 1a). If the words "biomarker and proteomics (or proteome)" are searched, we can also realize a massive number of articles in the past 13 years (30,719 articles), and an exponential increase in the number of lately published articles (Fig. 1b). Searching the word "biomarker" from 1996 to 2009 (1996 was when the first article of proteomics (or

D. Martins-de-Souza ( $₫)$

Max Planck Institute of Psychiatry, Proteomics and Biomarkers,

Munich, Germany

e-mail: martins@mpipsykl.mpg.de

D. Martins-de-Souza

Laboratório de Neurociências (LIM-27), Instituto Psiquiatria,

Faculdade de Medicina, Universidade de São Paulo, SP, Brazil proteome) and biomarker was published), we can find 342,751 articles, which means that proteomics research have published $8.96 \%$ of the articles which contains "biomarker" as keyword. This huge number of articles is very far to be at same proportion to the number of the recently discovered biomarkers that are useful to disease diagnosis, indicators of disease status, or targets to monitor and predict response to therapeutics or disease outcome and such data show us that a better definition for differentially expressed proteins with potential to be biomarkers has to be implemented, to leave the word "biomarker" to its own and well-defined meaning, avoiding the misuse of the term.

Recently, researchers have been used safer terms such as "potential biomarker" or "biomarker candidate" for proteins differentially expressed in diseased conditions that might have a potential as biomarker. I would propose that the correct term for most of developed research regarding biomarker discover based on the most of published articles not only for proteomics, but also in transcriptome and association studies, would be "potential biomarker candidate" (PBC). Why such name? Considering a differential proteome analysis comparing healthy and diseased tissue, differentially expressed protein will be found by high throughput and semi-automatic proteomics methods. At this point, I would say we would have a simple "biomarker candidate", which means something far, at least two levels away from a real biomarker. The biomarker candidates need to be validated in a greater and statistically significant universe of individual samples using distinct methodologies such as Western blot, ELISA or single and multiple reaction monitoring (SRM or MRM). If such proteins are indeed validated as differentially expressed, then we would have a second level of candidate, which I would call PBC. And we are clearly aware that for a $\mathrm{PBC}$ reach the 
Fig. 1 a "Biomarker" b

"Proteomic or proteomics or proteome and biomarker" on PubMed

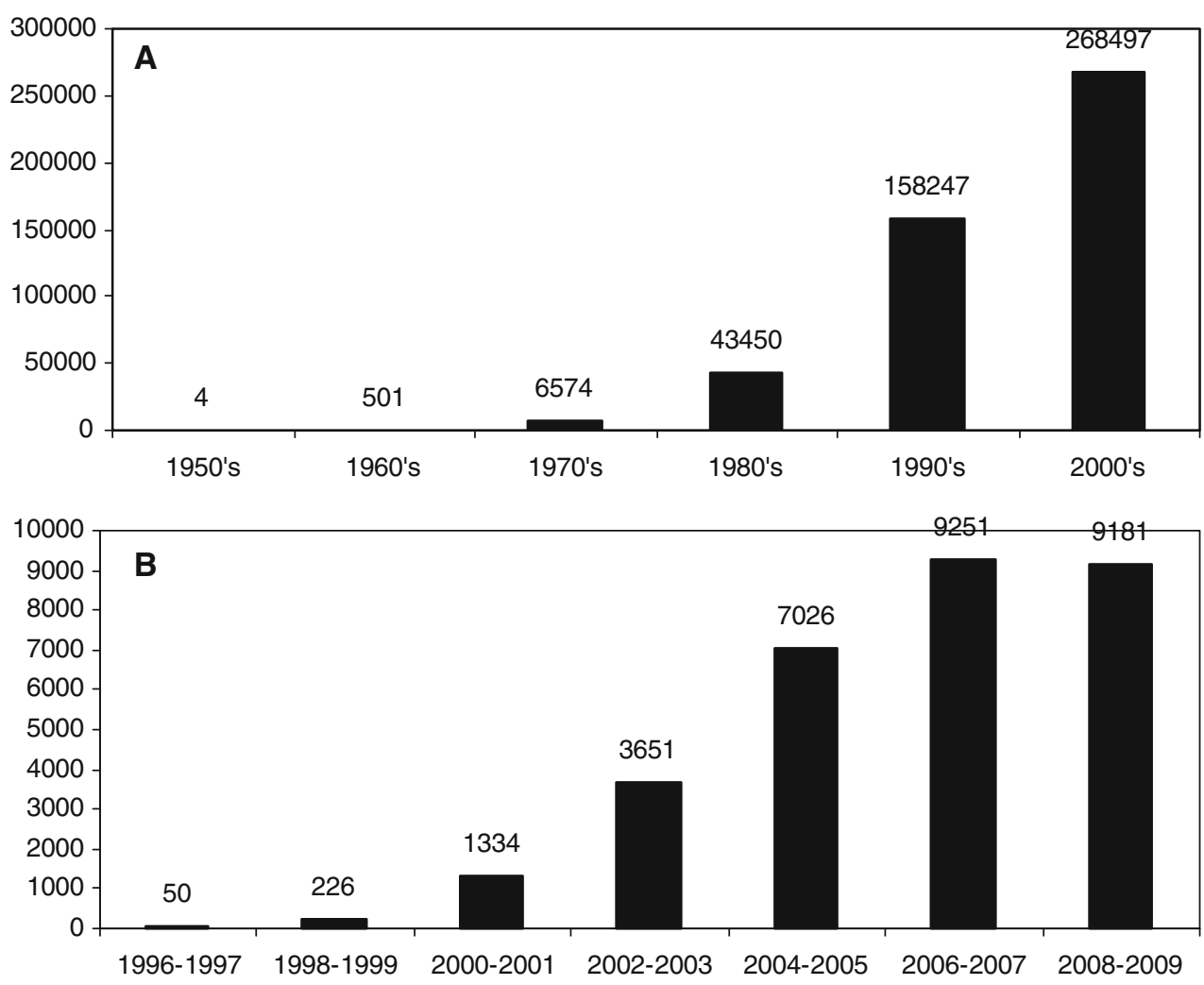

"biomarker" level, many other steps must be climbed, using different parameters and technologies, studying different sets of samples, considering potential confounding factors. A good review for that can be found at Mischak et al. [2] and its references.

Sets of adequate standards to proteomics studies for biomarker discovery have been already proposed, applied in proteome research [2] and required by proteomics journals. Now, we need to define to "potential biomarkers" or "biomarker candidates" a better and clearer term or name that could really be distinct from "biomarker", that has its own and well-defined meaning. For most of clinical proteomics studies, I believe that PBC would be a considerable suggestion. This way, it would be possible to clearly separate PBC from biomarker, two entities that have very different concepts and distinct values. Moreover, an adequate nomenclature would facilitate the judgment of the researchers and reviewers, who may feel more comfortable to read PBC instead biomarker in clinical proteomics studies.

Open Access This article is distributed under the terms of the Creative Commons Attribution Noncommercial License which permits any noncommercial use, distribution, and reproduction in any medium, provided the original author(s) and source are credited.

\section{References}

1. Chakravarty A (2003) Surrogate markers: their role in regulatory decision process. Food and Drug Administration, http://www.fda. gov/cder/Offices/Biostatistics/Chakravarty_376/sld016.htm

2. Mischak H, Apweiler R, Banks RE et al (2007) Clinical proteomics: a need to define the field and to begin to set adequate standards. Proteomics Clin Appl 1:148-156 